\begin{tabular}{|c|l|}
\hline Title & Markov property and strong additivity of von Neumann entropy for graded quantum systems \\
\hline Author(s) & Moriya, Hajime \\
\hline Citation & Journal of mathematical physics, 47(3), 033510 \\
\hline https:/doi.org/40.1063/2.2176911
\end{tabular}

Instructions for use 


\title{
Markov property and strong additivity of von Neumann entropy for graded quantum systems
}

\author{
Hajime Moriya \\ Department of Mathematics, Graduate School of Science, Hokkaido University Kita 10, \\ Nishi 8, Kita-Ku, Sapporo, Hokkaido, 060-0810, Japan
}

(Received 28 October 2005; accepted 24 January 2006; published online 27 March 2006)

\begin{abstract}
The quantum Markov property is equivalent to the strong additivity of von Neumann entropy for graded quantum systems. The additivity of von Neumann entropy for bipartite graded systems implies the statistical independence of states. However, the structure of Markov states for graded systems is different from that for tensorproduct systems which have trivial grading. For three-composed graded systems we have U(1)-gauge invariant Markov states whose restriction to the marginal pair of subsystems is nonseparable. (C) 2006 American Institute of Physics.
\end{abstract}

[DOI: $10.1063 / 1.2176911]$

\section{INTRODUCTION}

We are interested in characterization of state correlations for general composite systems which do not necessarily satisfy the local commutativity. Here we specifically consider finite-dimensional quantum systems with graded commutation relations. For such systems as well, we can divide the total system into the subsystem on a specified region and that on its complement region and discuss the nature of state correlations between them as in the tensor-product systems.

We have pointed out that known criterions of separability for tensor-product systems should be altered for lattice fermion systems ${ }^{11}$ when fermion hopping terms are present. (Note that purely fermionic correlation due to fermion hopping terms cannot be distilled to use.)

We are going to discuss quantum Markov property, ${ }^{1}$ a quantum version of Markov property invented by Accardi. This is given by means of quasiconditional expectations and has played various roles, see, e.g., Ref. 2. We can view the Markov property as a kind of characterization of state correlation for composite systems. A pivotal example of quantum composite systems is tensor product of Hilbert spaces for which lots of works, prominently those on Markov chains for one-dimensional quantum spin lattice systems have been done.

We note that the definition of Markov property has been given under a very general setting that is not limited to the most familiar case of tensor-product systems. That is, it does not require in principle any specific algebraic location among subsystems imbedded in the total system. ${ }^{3}$ Its detailed analysis for the setting of nonindependent systems, however, has started only recently. Reference 4 investigates Markov chains for one-dimensional (spinless) fermion lattice systems. It has been clarified there that the notion of Markov property and of the Markov chain is well applicable to fermion lattice systems. [More precisely, the above Markov chain refers to (d)-Markovian chain, ${ }^{1}$ see also Ref. 13 on the generalized Markov chain.] Furthermore, a class of $\mathrm{U}(1)$-gauge invariant Markov chains with fermionic hopping correlations is given. ${ }^{4}$

It has been shown that the Markov property is tightly related to the sufficiency of conditional expectations through the strong subadditivity of von Neumann entropy: A state of a threecomposed tensor-product system is Markovian if and only if it takes the equality for the strong subadditivity inequality of entropy, which will be referred to as "the strong additivity of entropy." $16,6,12,7$

We show that a similar equivalence relation of the Markov property and the strong additivity of entropy is valid for graded quantum systems. Its proof proceeds in much the same way as that 
for the tensor-product case following Ref. 15 (whose methods and results can be used in the present nonindependent situation) with some simple modifications due to the grading.

We now introduce the graded systems under our consideration. Let $\mathfrak{F}$ be a lattice and $\left\{\mathcal{A}_{\mathbf{I}} ; \mathbf{I}\right.$ $\in \mathfrak{F}\}$ be a family of $*$-algebras that have a common unital element denoted $\mathbf{1}$. If $\mathbf{I} \subset \mathbf{J}$, then $\mathcal{A}_{\mathbf{I}} \subset \mathcal{A}_{\mathbf{J}}$, and if $\mathbf{I} \cap \mathbf{J}=\varnothing$, then $\mathcal{A}_{\mathbf{I}} \cap \mathcal{A}_{\mathbf{J}}=\mathrm{C} \mathbf{1}$. Let $\Theta$ be an involutive $*$-automorphism of $\mathcal{A}$ that determines the grading as

$$
\mathcal{A}^{e}:=\{A \in \mathcal{A} \mid \Theta(A)=A\}, \quad \mathcal{A}^{o}:=\{A \in \mathcal{A} \mid \Theta(A)=-A\} .
$$

We assume that our grading transformation $\Theta$ is nontrivial. The above $\mathcal{A}^{e}$ and $\mathcal{A}^{o}$ (which is not empty) are called the even and odd parts of $\mathcal{A}$. For $\mathbf{I} \in \mathfrak{F}$

$$
\mathcal{A}_{\mathbf{I}}^{e}:=\mathcal{A}^{e} \cap \mathcal{A}_{\mathbf{I}}, \quad \mathcal{A}_{\mathbf{I}}^{o}:=\mathcal{A}^{o} \cap \mathcal{A}_{\mathbf{I}} .
$$

For $A \in \mathcal{A}\left(\mathcal{A}_{\mathbf{I}}\right)$ we have the even-odd decomposition:

$$
A=A_{+}+A_{-}, \quad A_{+}:=\frac{1}{2}(A+\Theta(A)) \in \mathcal{A}^{e}\left(\mathcal{A}_{\mathbf{I}}^{e}\right), \quad A_{-}:=\frac{1}{2}(A-\Theta(A)) \in \mathcal{A}^{o}\left(\mathcal{A}_{\mathbf{I}}^{o}\right) .
$$

We introduce $\mathrm{U}(1)$ gauge transformation:

$$
\gamma_{\theta}\left(a_{i}^{*}\right)=e^{i \theta} a_{i}^{*}, \quad \gamma_{\theta}\left(a_{i}\right)=e^{-i \theta} a_{i}
$$

for $\theta \in \mathbb{C}^{1}$. A state invariant under $\Theta$ is called even, and that invariant under $\gamma_{\theta}$ for any $\theta \in \mathbb{C}^{1}$ is called $\mathrm{U}(1)$-gauge invariant.

If a pair of subsets $\mathbf{I}$ and $\mathbf{J}$ of $\mathfrak{F}$ are disjoint, then the following graded commutation relations hold:

$$
\left[\mathcal{A}_{\mathbf{I}}^{e}, \mathcal{A}_{\mathbf{J}}^{e}\right]=0, \quad\left[\mathcal{A}_{\mathbf{I}}^{e}, \mathcal{A}_{\mathbf{J}}^{o}\right]=0, \quad\left[\mathcal{A}_{\mathbf{I}}^{o}, \mathcal{A}_{\mathbf{J}}^{e}\right]=0, \quad\left\{\mathcal{A}_{\mathbf{I}}^{o}, \mathcal{A}_{\mathbf{J}}^{o}\right\}=0,
$$

where $[A, B]=A B-B A$ denotes the commutator and $\{A, B\}=A B+B A$ the anticommutator.

We assume that $\mathcal{A}_{\mathrm{I}}$ is isomorphic to a finite-dimensional type-I factor (a full matrix algebra) for any $\mathbf{I} \in \mathfrak{F}$. Under this assumption, there is a unitary $v_{\mathbf{I}}$ in $\mathcal{A}_{\mathbf{I}}$ that implements $\Theta$ on $\mathcal{A}_{\mathbf{I}}$ as

$$
v_{\mathbf{I}}^{*}(A) v_{\mathbf{I}}=\Theta(A), \quad A \in \mathcal{A}_{\mathbf{I}} .
$$

This $v_{\mathbf{I}}$ is even, since $\Theta\left(v_{\mathbf{I}}\right)=v_{\mathbf{I}}^{*}\left(v_{\mathbf{I}}\right) v_{\mathbf{I}}=\left(v_{\mathbf{I}}^{*} v_{\mathbf{I}}\right) v_{\mathbf{I}}=v_{\mathbf{I}}$. For disjoint $\mathbf{I}$ and $\mathbf{J}$, the unitary $v_{\mathbf{I} \cup \mathbf{J}}$ of (6) for $\mathcal{A}_{\mathbf{I} \cup \mathbf{J}}$ is given by $v_{\mathbf{I}} v_{\mathbf{J}}$.

The lattice fermion system is a typical example of the graded quantum systems. Let $a_{i}^{*}$ and $a_{i}$ be creation and annihilation operators on the specified site $i$ in a lattice. For each finite subset $\mathbf{I}$, the subsystem $\mathcal{A}_{\mathbf{I}}$ are generated by $a_{i}^{*}$ and $a_{i}$ in $\mathbf{I}$. The even-odd grading transformation is given by

$$
\Theta\left(a_{i}^{*}\right)=-a_{i}^{*}, \quad \Theta\left(a_{i}\right)=-a_{i} .
$$

The unitary $v_{\mathbf{I}}$ is given by $v_{\mathbf{I}}:=\prod_{i \in \mathbf{I}} v_{i}, v_{i}:=a_{i}^{*} a_{i}-a_{i} a_{i}^{*}$.

We shall provide the plan of this paper. In Sec. II we introduce a formula of strong subadditivity of entropy $(\mathrm{SSA})^{8}$ in terms of the densities with respect to the tracial state for general composite systems made of finite-dimensional type-I factors. For the graded systems, it becomes the familiar formula of SSA in terms of the density matrices with respect to the matrix trace.

In Sec. III, the equivalence of the Markov property and the strong additivity of entropy for even states of the graded systems is shown. For noneven states, we have a weak result.

In Sec. IV, we consider restrictions of Markov states onto the marginal subsystems that are separated from each other. It was shown in Refs. 6 and 12 that a Markov state of a three-composed tensor-product system is separable (classically correlated) with respect to the marginal pair of subsystems. We show that this statement is invalid for the graded systems; there are $\mathrm{U}(1)$-gauge invariant (hence obviously even) Markov states that are nonseparable for the marginal pair. Intuitively speaking, such Markov states have fermion hopping correlations between the marginal subsystems. 
In Sec. $\mathrm{V}$, we show that a state of a graded bipartite system satisfies the additivity of von Neumann entropy if and only if it is a product state. This is almost obvious if the state under consideration is assumed to be even. The point is that the evenness (at least on one of the pair of subsystems) follows from the additivity of entropy.

\section{STRONG SUBADDITIVITY OF ENTROPY}

We provide the strong subadditivity of entropy for a general setting that encompasses nonindependent systems. Let $\mathcal{A}$ be a finite-dimensional type-I factor. Let $\tau$ denote the tracial state on $\mathcal{A}$. If an element $d \in \mathcal{A}$ is positive and normalized as $\tau(d)=1$, then it is called a density. For any state $\varphi$ of $\mathcal{A}$, there exists a unique density $\rho_{\varphi} \in \mathcal{A}$ called the density of $\varphi$ satisfying that

$$
\varphi(a)=\tau\left(\rho_{\varphi} a\right), \quad a \in \mathcal{A} .
$$

For the tracial state $\tau$, its density is obviously $\mathbf{1}$, the unity of $\mathcal{A}$.

Let $\rho_{1}$ and $\rho_{2}$ be a pair of densities of $\mathcal{A}$. The relative entropy for them is defined by

$$
H\left(\rho_{1}, \rho_{2}\right):=\tau\left(\rho_{1}\left(\ln \rho_{1}-\ln \rho_{2}\right)\right)
$$

if the support of $\rho_{1}$ is contained in $\rho_{2}$. Otherwise, we set it $+\infty$. For a pair of two states $\varphi$ and $\psi$ on $\mathcal{A}$, their relative entropy is

$$
H(\varphi, \psi):=H\left(\rho_{\varphi}, \rho_{\psi}\right)
$$

We define the entropy for a given state $\varphi$ as

$$
\hat{S}(\varphi):=-\varphi\left(\ln \rho_{\varphi}\right)
$$

We see

$$
\hat{S}(\varphi)=-H(\varphi, \tau)
$$

Probably the following is a more frequently used definition of entropy:

$$
S(\varphi):=-\operatorname{Tr}\left(D_{\varphi} \ln D_{\varphi}\right)=-\varphi\left(\ln D_{\varphi}\right),
$$

where $\operatorname{Tr}$ is the matrix trace that takes 1 for each one-dimensional projection, and $D_{\varphi}$ denotes the density matrix of $\varphi$ with respect to $\mathbf{T r}$. We see

$$
\hat{S}(\varphi)=S(\varphi)-S(\tau)=S(\varphi)-\ln \operatorname{Tr}(\mathbf{1})
$$

for any state $\varphi$. Hence if $\mathcal{A}$ is a $n$ by $n$ full matrix algebra, $n \in \mathbb{N}$, then the difference $S(\varphi)$ $-\hat{S}(\varphi)$ is constantly $\ln n$.

Let $\mathcal{B}$ be a subalgebra of $\mathcal{A}$. We denote the (uniquely determined) conditional expectation from $\mathcal{A}$ onto $\mathcal{B}$ with respect to the tracial state by $E_{\mathcal{B}}^{\mathcal{A}}$. Here, the upper-right subscript of $E$ indicates the domain and the lower-right the range. Let $\varphi_{\mathcal{B}}$ denote the restriction of $\varphi$ to $\mathcal{B}$. Then the density of $\varphi_{\mathcal{B}}$ is given by that of $\varphi$ as

$$
\rho_{\varphi_{\mathcal{B}}}=E_{\mathcal{B}}^{\mathcal{A}}\left(\rho_{\varphi}\right)
$$

We have

$$
\hat{S}\left(\varphi_{\mathcal{B}}\right)-\hat{S}(\varphi)=H\left(\rho_{\varphi}, \rho_{\varphi_{\mathcal{B}}}\right)=H\left(\varphi,\left.\varphi_{\mathcal{B}} \otimes \operatorname{tr}\right|_{\mathcal{A} \cap \mathcal{B}^{\prime}}\right)=H\left(\varphi, \varphi \circ E_{\mathcal{B}}^{\mathcal{A}}\right) .
$$

As a special case of (12),

$$
\hat{S}\left(\varphi_{\mathcal{B}}\right)=\hat{S}\left(\varphi \circ E_{\mathcal{B}}^{\mathcal{A}}\right)
$$


In fact, we have so far assumed that $\varphi$ is a faithful state, but (12) is valid when $\varphi$ is nonfaithful. To see this, we take $\varepsilon \cdot \tau+(1-\varepsilon) \varphi$ where $\varepsilon$ is a positive small number and then take the limit $\varepsilon \rightarrow 0$.

Let us take three disjoint subsets $A, B$, and $C$. Let $\mathcal{A}_{A B C}, \mathcal{A}_{A B}, \mathcal{A}_{B C}$, and $\mathcal{A}_{B}$ denote finitedimensional quantum systems corresponding to the indexes. Let $E_{A, B}^{A, B, C}$ and $E_{A}^{A, B}$ denote the trace preserving conditional expectation from $\mathcal{A}_{A B C}$ onto $\mathcal{A}_{A B}$ and that from $\mathcal{A}_{A B}$ onto $\mathcal{A}_{A}$, respectively. We use similar notations for other indexes. If the domain is the total system $\mathcal{A}_{A B C}$, then we simply write, e.g., $E_{A, B}$ instead of $E_{A, B}^{A, B, C}$ when there is no fear of confusion.

The following five conditions, called the commuting square condition, are all equivalent to each other:

(2) $E_{B, C} \mid \mathcal{A}_{A B}=E_{B}^{A, B}$

(3) $\mathcal{A}_{B}=\mathcal{A}_{A B} \cap \mathcal{A}_{B C}$ and $E_{A, B} E_{B, C}=E_{B, C} E_{A, B}$,

(4) $E_{A, B} E_{B, C}=E_{B}$,

(5) $E_{B, C} E_{A, B}=E_{B}$.

If our three-composed system $\mathcal{A}_{A B C}$ satisfies this commuting square condition, then the strong subadditivity of entropy $\hat{S}(\psi)$ for any state $\psi$ follows. The proof is standard and easy, but we recapture it for completeness.

Proposition 1: Let $\mathcal{A}_{A B C}, \mathcal{A}_{A B}, \mathcal{A}_{B C}$, and $\mathcal{A}_{B}$ be finite-dimensional factors satisfying the commuting square condition, and let $\psi_{A B C}$ be an arbitrary state on $\mathcal{A}_{A B C}$. Then

$$
\hat{S}\left(\psi_{A B C}\right)-\hat{S}\left(\psi_{A B}\right)-\hat{S}\left(\psi_{B C}\right)+\hat{S}\left(\psi_{B}\right) \leqslant 0 .
$$

Furthermore, if the system satisfies the graded commutation relations (5), then

$$
S\left(\psi_{A B C}\right)-S\left(\psi_{A B}\right)-S\left(\psi_{B C}\right)+S\left(\psi_{B}\right) \leqslant 0 .
$$

Proof: By (12) and (13), and the relation $E_{B, C} E_{A, B}=E_{A, B} E_{B, C}=E_{B}$, we obtain

$$
\begin{aligned}
\hat{S}\left(\psi_{B C}\right)-\hat{S}\left(\psi_{A B C}\right) & =H\left(\psi_{A B C}, \psi_{A B C} \circ E_{B, C}\right) \geqslant H\left(\psi_{A B C} \circ E_{A, B}, \psi_{A B C} \circ E_{B, C} \circ E_{A, B}\right) \\
& =H\left(\psi_{A B C} \circ E_{A, B}, \psi_{A B C} \circ E_{A, B} \circ E_{B, C}\right)=H\left(\psi_{A B C} \circ E_{A, B}, \psi_{A B C} \circ E_{B}\right) \\
& =\hat{S}\left(\psi_{A B C} \circ E_{B}\right)-\hat{S}\left(\psi_{A B C} \circ E_{A, B}\right)=\hat{S}\left(\psi_{B}\right)-\hat{S}\left(\psi_{A B}\right),
\end{aligned}
$$

where the inequality is due to the monotonicity of relative entropy under the action of completely positive maps.

Let us turn to the graded systems of finite-dimensional factors, which satisfy the commuting square condition. ${ }^{5}$ Suppose that $\mathbf{I}$ and $\mathbf{J}$ are disjoint subsets. Then the matrix trace on $\mathcal{A}_{\mathbf{I} \cup \mathbf{J}}$ denoted $\mathbf{T r}_{\mathbf{I} \cup \mathbf{J}}$ is given by the product extension of those in $\mathcal{A}_{\mathbf{I}}$ and in $\mathcal{A}_{\mathbf{J}}$ denoted $\mathbf{T r}_{\mathbf{I}}$ and $\mathbf{T r}_{\mathbf{J}}$, respectively. Thus we have $\mathbf{T r}_{\mathbf{I} \cup \mathbf{J}}(\mathbf{1})=\mathbf{T r}_{\mathbf{I}}(\mathbf{1}) \times \mathbf{T r}(\mathbf{1})$. Now (14) and (10) imply (15).

As this proposition indicates, the strong additivity of entropy is satisfied irrespective of whether states are even or not. In Refs. 9 and 10 we have shown that noneven states may induce pathological state correlations and some entropy inequalities known for tensor-product systems do not hold in general for the graded systems.

\section{MARKOV PROPERTY AND STRONG ADDITIVITY}

It is obvious that the equality of (14) and of (15) is equivalent to that of (16), i.e.,

$$
H\left(\psi_{A B C}, \psi_{A B C} \circ E_{B, C}\right)=H\left(\psi_{A B C} \circ E_{A, B}, \psi_{A B C} \circ E_{B}\right)
$$

equivalently,

$$
H\left(\rho_{\psi_{A B C}}, \rho_{\psi_{B C}}\right)=H\left(\rho_{\psi_{A B}}, \rho_{\psi_{B}}\right) .
$$


By a general result of the sufficiency given in Ref. 15, (17) implies that the conditional expectation $E_{A, B}$ is sufficient for $\psi_{A B C}{ }^{\circ} E_{B, C}$ and $\psi_{A B C}$, that is, there exists a completely positive map that recovers $\psi_{A B C}{ }^{\circ} E_{B, C}$ and $\psi_{A B C}$ from $\psi_{A B C}{ }^{\circ} E_{B, C}{ }^{\circ} E_{A, B}$ and $\psi_{A B C}{ }^{\circ} E_{A, B}$, respectively. The canonical form of such maps is given as follows. ${ }^{12}$

Let $\alpha$ denote the completely positive map on $\mathcal{A}$ defined by

$$
\alpha(X):=\rho_{\psi_{B}}{ }^{-1 / 2} E_{A, B}\left(\rho_{\psi_{B C}}{ }^{1 / 2} X \rho_{\psi_{B C}}{ }^{1 / 2}\right) \rho_{\psi_{B}}{ }^{-1 / 2}, \quad X \in \mathcal{A}_{A B C} .
$$

Let $T^{\sharp}$ denote the dual of $\alpha$ with respect to the tracial stare, which is written as

$$
T^{\sharp}(X):=\rho_{\psi_{B C}}{ }^{1 / 2} \rho_{\psi_{B}}{ }^{-1 / 2} X \rho_{\psi_{B}}{ }^{-1 / 2} \rho_{\psi_{B C}}{ }^{1 / 2}, \quad X \in \alpha\left(\mathcal{A}_{A B C}\right) .
$$

It is easy to see $T^{\sharp}\left(\rho_{\psi_{B}}\right)=\rho_{\psi_{B C}}$. Also $T^{\sharp}\left(\rho_{\psi_{A B}}\right)=\rho_{\psi_{A B C}}$ is satisfied if and only if $E_{A, B}$ is sufficient for the given pair of states $\psi_{A B C}$ and $\psi_{A B C}{ }^{\circ} E_{B, C}$, equivalently, (17) holds.

The following is a more or less summary of the contents stated above. It corresponds to Theorem 5.2 of Ref. 16 and also Sec. V of Ref. 12 where the statement is for the tensor-product systems.

Proposition 2: Let $\mathcal{A}_{A B C}, \mathcal{A}_{A B}, \mathcal{A}_{B C}$, and $\mathcal{A}_{B}$ be finite-dimensional factors satisfying the commuting square condition. Let $\psi_{A B C}$ be an arbitrary faithful state on $\mathcal{A}_{A B C}$. The strong additivity of von Neumann entropy, i.e.,

$$
S\left(\psi_{A B C}\right)-S\left(\psi_{A B}\right)-S\left(\psi_{B C}\right)+S\left(\psi_{B}\right)=0
$$

is satisfied if and only if $E_{A, B}$ is sufficient for the pair of states $\psi_{A B C}$ and $\psi_{A B C}{ }^{\circ} E_{B, C}$. Let $\alpha$ denote the $\psi_{A B C}$-preserving (and $\psi_{A B C}{ }^{\circ} E_{B, C}$-preserving) conditional expectation from $\mathcal{A}_{A B C}$ to $\mathcal{A}_{A B}$ given as (19). Let $T^{\sharp}$ denote the dual of this $\alpha$ with respect to the tracial state whose concrete formula is given as (20). This $T^{\#}$ gives the canonical left inverse of $E_{A, B}$ for the densities of $\psi_{A B C}$ and $\psi_{A B C}{ }^{\circ} E_{B, C}$, that is,

$$
T^{\sharp}\left(\rho_{\psi_{B}}\right)=\rho_{\psi_{B C}}
$$

and

$$
T^{\sharp}\left(\rho_{\psi_{A B}}\right)=\rho_{\psi_{A B C}} .
$$

The set of fixed points of $\alpha$ contains $\mathcal{A}_{A}^{e}$. If the state $\psi_{A B C}$ is even, then the set of fixed points of $\alpha$ contains $\mathcal{A}_{A}$ and accordingly the Markov property of $\psi_{A B C}$ with respect to a triplet $\left(\mathcal{A}_{A}, \mathcal{A}_{B}, \mathcal{A}_{C}\right)$ is satisfied.

Proof: We shall confirm the part about the fixed point elements of $\alpha$. Take $X \in \mathcal{A}_{A}^{e}$, which is in the commutant of $\mathcal{A}_{B C}$. We have

$$
\begin{aligned}
\alpha(X) & =\rho_{\psi_{B}}^{-1 / 2} E_{A, B}\left(\rho_{\psi_{B C}}^{1 / 2} X \rho_{\psi_{B C}}^{1 / 2}\right) \rho_{\psi_{B}}^{-1 / 2}=\rho_{\psi_{B}}^{-1 / 2} E_{A, B}\left(X \rho_{\psi_{B C}}\right) \rho_{\psi_{B}}^{-1 / 2}=\rho_{\psi_{B}}^{-1 / 2} X E_{A, B}\left(\rho_{\psi_{B C}}\right) \rho_{\psi_{B}}^{-1 / 2} \\
& =\rho_{\psi_{B}}^{-1 / 2} X E_{A, B}\left(E_{B, C}\left(\rho_{\psi_{B C}}\right)\right) \rho_{\psi_{B}}^{-1 / 2}=\rho_{\psi_{B}}^{-1 / 2} X E_{B}\left(\rho_{\psi_{B C}}\right) \rho_{\psi_{B}}^{-1 / 2}=\rho_{\psi_{B}}^{-1 / 2} X \rho_{\psi_{B}} \rho_{\psi_{B}}^{-1 / 2}=X \rho_{\psi_{B}}^{-1 / 2} \rho_{\psi_{B}} \rho_{\psi_{B}}^{-1 / 2}=X .
\end{aligned}
$$

Suppose now that $\psi_{A B C}$ is even. Then $\rho_{\psi_{B C}} \in \mathcal{A}_{B C}^{e}$ and also $\rho_{\psi_{B}} \in \mathcal{A}_{B}^{e}$ commute with any $X$ $\in \mathcal{A}_{A}$. Hence we see that the above set of equalities (24) holds for this case.

From this result, if an even state satisfies the strong additivity of entropy, then the Markov property with respect to a triplet $\left(\mathcal{A}_{A}, \mathcal{A}_{B}, \mathcal{A}_{C}\right)$ in the sense of Ref. 1 (cf. Lemma 11.3 of Ref. 14) is satisfied. This in fact precisely specifies what we mean by the Markov property. 


\section{MARKOV STATES ON THE MARGINAL SUBSYSTEMS}

The definition of separable states (i.e., classically correlated states) for nonindependent systems is much the same as that for the tensor-product systems. ${ }^{11}$ That is, if a state is written as a convex sum of some product states, then it is called a separable state. Let $A$ and $C$ be a pair of disjoint subsets, and $\omega$ be a state on $\mathcal{A}_{A C}$. If

$$
\omega(X Y)=\omega(X) \omega(Y)
$$

for all $X \in \mathcal{A}_{A}$ and $Y \in \mathcal{A}_{C}$, then $\omega$ is called a product state with respect to the pair $\left(\mathcal{A}_{A}, \mathcal{A}_{C}\right)$. It is easy to see that the product property in the converse order,

$$
\omega(Y X)=\omega(Y) \omega(X)=\omega(X Y)
$$

follows from (25) and the graded commutation relations.

We discuss the property of Markov states with respect to $\left(\mathcal{A}_{A}, \mathcal{A}_{B}, \mathcal{A}_{C}\right)$ for the marginal subsystem $\mathcal{A}_{A C}$. As we announced in the introduction, Corollary 7 of Ref. 6 is invalid for the graded systems.

Proposition 3: For a three-composed graded system $\left(\mathcal{A}_{A}, \mathcal{A}_{B}, \mathcal{A}_{C}\right)$, there exist $U(1)$-gauge invariant states that satisfy the Markov property for $\left(\mathcal{A}_{A}, \mathcal{A}_{B}, \mathcal{A}_{C}\right)$ but are nonseparable for $\left(\mathcal{A}_{A}, \mathcal{A}_{C}\right)$.

We shall construct such Markov states. Using the Jordan-Wigner transformation, we set a three-composed tensor-product system in the following way. Let $v_{A}, v_{B}$, and $v_{A, B}$ denote the unitaries implementing $\Theta$ on the specified subsystems. Let $\mathcal{A}_{A}^{s}:=\mathcal{A}_{A}, \mathcal{A}_{A B}^{s}:=\mathcal{A}_{A B}, \mathcal{A}_{A B C}^{s}:=\mathcal{A}_{A B C}$, $\mathcal{A}_{B}^{s}:=\left\{\mathcal{A}_{B}^{e}, v_{A} \mathcal{A}_{B}^{o}\right\}, \mathcal{A}_{C}^{s}:=\left\{\mathcal{A}_{C}^{e}, v_{A, B} \mathcal{A}_{C}^{o}\right\}$, and $\mathcal{A}_{B C}^{s}:=\left\{\mathcal{A}_{B C}^{e}, v_{A} \mathcal{A}_{B C}^{o}\right\}$ where the notation $\{$,$\} denotes the$ algebra generated by the arguments. They induce a tensor-product system $\mathcal{A}_{A B C}^{s}=\mathcal{A}_{A}^{s} \otimes \mathcal{A}_{B}^{s} \otimes \mathcal{A}_{C}^{s}$. We assign finite-dimensional Hilbert spaces $\mathcal{H}_{A}^{-}, \mathcal{H}_{B}^{-}$and $\mathcal{H}_{C}^{-}$to $\mathcal{A}_{A}^{s}, \mathcal{A}_{B}^{s}$ and $\mathcal{A}_{C}^{s}$, respectively. We will use the next lemma later. Its proof is obvious.

Lemma 4: Let $\psi_{A B C}$ be an arbitrary even state on $\mathcal{A}_{A B C}$. It satisfies

$$
S\left(\psi_{A B C}\right)-S\left(\psi_{A B}\right)-S\left(\psi_{B C}\right)+S\left(\psi_{B}\right)=0,
$$

if and only if

$$
S\left(\psi_{A B C}\right)-S\left(\left.\psi\right|_{\mathcal{A}_{A B}^{s}}\right)-S\left(\left.\psi\right|_{\mathcal{A}_{B C}^{s}}\right)+S\left(\left.\psi\right|_{\mathcal{A}_{B}^{s}}\right)=0 .
$$

For a while we will focus on the two composed system, $\mathcal{A}_{A C}$. In Ref. 11 we have discussed how the state correlation (separability, nonseparability) will remain or change under the JordanWigner transformation which maps the CAR pair $\left(\mathcal{A}_{A}, \mathcal{A}_{C}\right)$ to $\left(\mathcal{A}_{A}, \mathcal{A}_{C}^{\widetilde{s}}\right)$, where $\mathcal{A}_{C}^{\widetilde{s}}$ denotes the commutant of $\mathcal{A}_{A}$ in $\mathcal{A}_{A C}$ and is explicitly given as $\left\{\mathcal{A}_{C}^{e}, v_{A} \mathcal{A}_{C}^{o}\right\}$. (Note that $\mathcal{A}_{C}^{\tilde{s}}$ is different from previously introduced $\mathcal{A}_{C}^{s}$.) It has been shown that the set of all separable states for the CAR pair is strictly smaller than that for the tensor-product pair. That is, if $\omega_{A C}$ is a separable state for the pair $\left(\mathcal{A}_{A}, \mathcal{A}_{C}\right)$, then so it is for $\left(\mathcal{A}_{A}, \mathcal{A}_{C}^{\widetilde{s}}\right)$. However, there exist $\mathrm{U}(1)$-invariant states that are separable for the latter but nonseparable for the former. We introduce an example of such states from Ref. 11.

Let $k_{A}$ and $k_{C}$ be some nonzero odd elements in $\mathcal{A}_{A}$ and in $\mathcal{A}_{C}$, e.g., field operators on specified regions. Let $K:=1 / 2\left(k_{A}^{*} k_{C}-k_{A} k_{C}^{*}\right)$ which is self-adjoint and denotes fermion-hopping interaction between $\mathcal{A}_{A}$ and $\mathcal{A}_{C}$. Suppose that $\left\|k_{A}\right\| \leqslant 1\left\|k_{C}\right\| \leqslant 1$, then $\|K\| \leqslant 1$. For $\lambda \in \mathbb{R},|\lambda| \leqslant 1$, $\rho_{A C, \lambda}:=1+\lambda K$ gives a density operator. For $0<|\lambda| \leqslant 1$, the state on $\mathcal{A}_{A C}$ with its density $\rho_{A C, \lambda}$ gives a state satisfying all the desired conditions.

Now take such a $\mathrm{U}(1)$-gauge invariant state $\omega_{A C}$ on $\mathcal{A}_{A C}$. It has a state decomposition $\omega_{A C}$ $=\sum_{i=1}^{n} \lambda_{i} \omega_{A C, i}, 0<\lambda_{i}<1, \Sigma \lambda_{i}=1$, such that each $\omega_{A C, i}$ is a product state for $\left(\mathcal{A}_{A}, \mathcal{A}_{C}^{\tilde{s}}\right)$, but has no product-state decomposition for $\left(\mathcal{A}_{A}, \mathcal{A}_{C}\right)$. From this, we are going to construct a state on $\mathcal{A}_{A B C}$ that proves Proposition 3.

Let us assume that the dimension of $\mathcal{H}_{B}^{-}$is equal or more than $n$. Then we have a set of $n$ non-zero even orthogonal projections $p_{i} \in \mathcal{A}_{B}^{e}, 1 \leqslant i \leqslant n$. Let $\omega_{B, i}(X):=\tau\left(p_{i} X\right) / \tau\left(p_{i}\right)$, for $X \in \mathcal{A}_{B}$. 
Those are all even states of $\mathcal{A}_{B}$. Let $\omega_{A B C}:=\sum_{i=1}^{n} \lambda_{i} \omega_{A C, i}{ }^{\circ} \omega_{B, i}$, where $\omega_{A C, i^{\circ}} \omega_{B, i}$ denotes the (uniquely determined) product state extension of $\omega_{A C, i}$ on $\mathcal{A}_{A C}$ and $\omega_{B, i}$ on $\mathcal{A}_{B}$, see Ref. 5.

We will see that $\omega_{A C, i}{ }^{\circ} \omega_{B, i}$ gives a product state for $\left(\mathcal{A}_{A}, \mathcal{A}_{C}^{s}\right)$ when restricted to $\mathcal{A}_{A} \otimes \mathcal{A}_{C}^{s}$. We must check this for the product element $a c_{+}$such that $a \in \mathcal{A}_{A}$ and $c_{+} \in \mathcal{A}_{C}^{e}$, and for $a\left(v_{A} v_{B} c_{-}\right)$such that $a \in \mathcal{A}_{A}$ and $c_{-} \in \mathcal{A}_{C}^{o}$. We have

$$
\omega_{A C, i} \circ \omega_{B, i}\left(a c_{+}\right)=\omega_{A C, i}\left(a c_{+}\right)=\omega_{A C, i}(a) \omega_{A C, i}\left(c_{+}\right)=\omega_{A C, i} \circ \omega_{B, i}(a) \omega_{A C, i} \circ \omega_{B, i}\left(c_{+}\right),
$$

and using the product property of $\omega_{A C, i}$ for $\left(\mathcal{A}_{A}, \mathcal{A}_{C}^{\tilde{s}}\right)$,

$$
\begin{gathered}
\omega_{A C, i} \circ \omega_{B, i}\left(a v_{A} v_{B} c_{-}\right)=\omega_{A C, i} \circ \omega_{B, i}\left(a v_{A} c_{-} v_{B}\right)=\omega_{A C, i}\left(a v_{A} c_{-}\right) \omega_{B, i}\left(v_{B}\right)=\omega_{A C, i}(a) \omega_{A C, i}\left(v_{A} c_{-}\right) \omega_{B, i}\left(v_{B}\right) \\
=\omega_{A C, i}(a) \omega_{A C, i} \circ \omega_{B, i}\left(v_{A} c_{-} v_{B}\right)=\omega_{A C, i} \circ \omega_{B, i}(a) \omega_{A C, i} \circ \omega_{B, i}\left(v_{A} v_{B} c_{-}\right) .
\end{gathered}
$$

Hence, $\omega_{A C, i}{ }^{\circ} \omega_{B, i}$ has a product state restriction, and accordingly $\omega_{A B C}$ has a separable state restriction for $\left(\mathcal{A}_{A}, \mathcal{A}_{C}^{s}\right)$. We conclude that our $\omega_{A B C}$ has the structure as in Theorem 6 of Ref. 6 or as the formula (14) of Ref. 12 with respect to $\left(\mathcal{H}_{\bar{A}}^{-}, \mathcal{H}_{\bar{B}}, \mathcal{H}_{\bar{C}}\right)$. Hence, it satisfies the Markov property with respect to $\left(\mathcal{A}_{A}^{s}, \mathcal{A}_{B}^{s}, \mathcal{A}_{C}^{s}\right)$.

From the equivalence of the Markov property and the strong additivity of entropy for threecomposed tensor-product systems, which has been shown in the above references, (28) is satisfied for $\omega_{A B C}$. Since it is even, it satisfies (27) as well and, hence, is Markovian with respect to $\left(\mathcal{A}_{A}^{s}, \mathcal{A}_{B}^{s}, \mathcal{A}_{C}^{s}\right)$ by Proposition 2. As $\left.\omega_{A C}\right|_{\mathcal{A}_{A C}}=\omega_{A C}$ is obviously nonseparable for $\left(\mathcal{A}_{A}, \mathcal{A}_{C}\right)$ by definition, $\omega_{A B C}$ gives a state showing Proposition 3.

\section{ADDITIVITY OF VON NEUMANN ENTROPY AND THE PRODUCT PROPERTY}

In this section, we consider a two-composed graded system $\mathcal{A}_{A C}$ generated by $\mathcal{A}_{A}$ and $\mathcal{A}_{C}$. Namely, we treat the case where the intersection region $B$ is trivial. Then the strong subadditivity of entropy (15) becomes

$$
S\left(\psi_{A C}\right)-S\left(\psi_{A}\right)-S\left(\psi_{C}\right) \leqslant 0,
$$

which is called the subadditivity of entropy. We discuss characterization of additivity of entropy, i.e., the condition of equality of this inequality.

The answer is very simple for tensor-product systems: a state satisfies the additivity of entropy if and only if it is a product state. For the graded system, we can show a similar result easily under the assumption that the marginal states $\psi_{A}$ and $\psi_{C}$ are not both noneven. Let $\psi_{A} \circ \psi_{C}$ denote the product state of $\mathcal{A}_{A C}$ whose restrictions to $\mathcal{A}_{A}$ and $\mathcal{A}_{C}$ are $\psi_{A}$ and $\psi_{C}$. Its existence is guaranteed if $\psi_{A}$ and/or $\psi_{C}$ is even. Then we have

$$
S\left(\psi_{A C}\right)-S\left(\psi_{A}\right)-S\left(\psi_{C}\right)=-H\left(\psi_{A C}, \psi_{A} \circ \psi_{C}\right) \leqslant 0 .
$$

By the strict positivity of relative entropy, it is 0 if and only if $\psi_{A C}=\psi_{A}^{\circ} \psi_{C}$.

Now we drop the evenness assumption on the states. If $\psi_{A}$ and $\psi_{C}$ are both noneven, then there is no product state extension for them. ${ }^{5}$ Hence, the above argument using the strict positivity of relative entropy does not work for the general case.

Using Ref 12 we obtain the following result.

Proposition 5: Let $\psi_{A C}$ be a state of the two-composed graded system $\mathcal{A}_{A C}$. It satisfies the additivity of entropy

$$
S\left(\psi_{A C}\right)-S\left(\psi_{A}\right)-S\left(\psi_{C}\right)=0,
$$

if and only if it is a product state for $\left(\mathcal{A}_{A}, \mathcal{A}_{C}\right)$. If it is the case, at least one of $\psi_{A}$ and $\psi_{C}$ is even.

Proof: The equivalence of (33) and (18) when the middle part $B$ is empty implies that (33) is equivalent to 


$$
H\left(\rho_{\psi_{A C}}, \rho_{\psi_{C}}\right)=H\left(\rho_{\psi_{A}}, \mathbf{1}\right) .
$$

This is equivalent to say that $E_{A}$ is sufficient for $\rho_{\psi_{A C}}$ and $\rho_{\psi_{C}}$. Now from (20) the canonical left inverse of $E_{A}$ for those densities is given by

$$
T^{\sharp}(X):=\rho_{\psi_{C}}^{1 / 2} X \rho_{\psi_{C}}^{1 / 2}, \quad X \in \mathcal{A}_{A} .
$$

Hence we have

$$
\rho_{\psi_{A C}}=T^{\sharp}\left(\rho_{\psi_{A}}\right)=\rho_{\psi_{C}}^{1 / 2} \rho_{\psi_{A}} \rho_{\psi_{C}}^{1 / 2} .
$$

Exchanging $A$ and $C$ and repeating the same argument as above, we have also

$$
\rho_{\psi_{A C}}=\rho_{\psi_{A}}^{1 / 2} \rho_{\psi_{C}} \rho_{\psi_{A}}^{1 / 2} .
$$

Let us take the decomposition of $\rho_{\psi_{A}}$ into its even-odd parts and that of $\rho_{\psi_{C}}$ as in (3),

$$
\begin{aligned}
& \rho_{\psi_{A}}=\rho_{\psi_{A^{+}}}+\rho_{\psi_{A^{-}},}, \rho_{\psi_{A^{+}}} \in \mathcal{A}_{A}^{e}, \quad \rho_{\psi_{A^{-}}} \in \mathcal{A}_{A}^{o}, \\
& \rho_{\psi_{C}}=\rho_{\psi_{C^{+}}}+\rho_{\psi_{C^{-}}}, \quad \rho_{\psi_{C^{+}}} \in \mathcal{A}_{C}^{e}, \quad \rho_{\psi_{C^{-}}} \in \mathcal{A}_{C}^{o} .
\end{aligned}
$$

Similarly take the even-odd decomposition of $\rho_{\psi_{A}}^{1 / 2}$ and that of $\rho_{\psi_{C}}^{1 / 2}$ in the following:

$$
\begin{aligned}
& \rho_{\psi_{A}}^{1 / 2}=a_{+}+a_{-}, \quad a_{+} \in \mathcal{A}_{A}^{e}, \quad a_{-} \in \mathcal{A}_{A}^{o}, \\
& \rho_{\psi_{C}}^{1 / 2}=c_{+}+c_{-}, \quad c_{+} \in \mathcal{A}_{C}^{e}, \quad c_{-} \in \mathcal{A}_{C}^{o} .
\end{aligned}
$$

Since the densities are positive hence self-adjoint, each of $a_{+}, a_{-}, c_{+}$, and $c_{-}$is self-adjoint. We have

$$
\begin{gathered}
\rho_{\psi_{A}}=\left(\rho_{\psi_{A}}^{1 / 2}\right)^{2}=a_{+}^{2}+a_{-}^{2}+a_{+} a_{-}+a_{-} a_{+}, \\
\rho_{\psi_{A^{+}}}=a_{+}^{2}+a_{-}^{2}, \\
\rho_{\psi_{A^{-}}}=a_{+} a_{-}+a_{-} a_{+},
\end{gathered}
$$

and

$$
\begin{gathered}
\rho_{\psi_{C}}=c_{+}^{2}+c_{-}^{2}+c_{+} c_{-}+c_{-} c_{+}, \\
\rho_{\psi_{A^{+}}}=c_{+}^{2}+c_{-}^{2}, \\
\rho_{\psi_{A^{-}}}=c_{+} c_{-}+c_{-} c_{+} .
\end{gathered}
$$

Now we shall express the equality $\rho_{\psi_{C}}^{1 / 2} \rho_{\psi_{A}} \rho_{\psi_{C}}^{1 / 2}=\rho_{\psi_{A}}^{1 / 2} \rho_{\psi_{C}} \rho_{\psi_{A}}^{1 / 2}=\rho_{\psi_{A C}}$ in terms of $a_{+}, a_{-}, c_{+}$, and $c_{-}$. We compute

$$
\begin{aligned}
\rho_{\psi_{C}}^{1 / 2} \rho_{\psi_{A}} \rho_{\psi_{C}}^{1 / 2} & =\rho_{\psi_{C}}^{1 / 2}\left(\rho_{\psi_{A^{+}}}+\rho_{\psi_{A^{-}}}\right) \rho_{\psi_{C}}^{1 / 2}=\left(\rho_{\psi_{A}+} \rho_{\psi_{C}}^{1 / 2}+\rho_{\psi_{A^{-}}} \Theta\left(\rho_{\psi_{C}}^{1 / 2}\right)\right) \rho_{\psi_{C}}^{1 / 2}=\rho_{\psi_{A}+} \rho_{\psi_{C}}+\rho_{\psi_{A}-} \Theta\left(\rho_{\psi_{C}}^{1 / 2}\right) \rho_{\psi_{C}}^{1 / 2} \\
& =\left(a_{+}^{2}+a_{-}^{2}\right)\left(c_{+}^{2}+c_{-}^{2}+c_{+} c_{-}+c_{-} c_{+}\right)+\left(a_{+} a_{-}+a_{-} a_{+}\right)\left(c_{+}-c_{-}\right)\left(c_{+}+c_{-}\right) \\
& =a_{+}^{2}\left(c_{+}^{2}+c_{-}^{2}+c_{+} c_{-}+c_{-} c_{+}\right)+a_{-}^{2}\left(c_{+}^{2}+c_{-}^{2}+c_{+} c_{-}+c_{-} c_{+}\right)+a_{+} a_{-}\left(c_{+}^{2}-c_{-}^{2}-c_{-} c_{+}+c_{+} c_{-}\right)
\end{aligned}
$$




$$
+a_{-} a_{+}\left(c_{+}^{2}-c_{-}^{2}-c_{-} c_{+}+c_{+} c_{-}\right) \text {. }
$$

Also,

$$
\begin{aligned}
\rho_{\psi_{A}}^{1 / 2} \rho_{\psi_{C}} \rho_{\psi_{A}}^{1 / 2}= & \rho_{\psi_{A}}^{1 / 2}\left(\rho_{\psi_{C^{+}}}+\rho_{\psi_{C^{-}}}\right) \rho_{\psi_{A}}^{1 / 2}=\rho_{\psi_{A}} \rho_{\psi_{C^{+}}}+\rho_{\psi_{A}}^{1 / 2} \Theta\left(\rho_{\psi_{A}}^{1 / 2}\right) \rho_{\psi_{C^{-}}} \\
= & \left(a_{+}^{2}+a_{-}^{2}+a_{+} a_{-}+a_{-} a_{+}\right)\left(c_{+}^{2}+c_{-}^{2}\right)+\left(a_{+}^{2}-a_{-}^{2}-a_{+} a_{-}+a_{-} a_{+}\right)\left(c_{+} c_{-}+c_{-} c_{+}\right) \\
= & a_{+}^{2}\left(c_{+}^{2}+c_{-}^{2}+c_{+} c_{-}+c_{-} c_{+}\right)+a_{-}^{2}\left(c_{+}^{2}+c_{-}^{2}-c_{+} c_{-}-c_{-} c_{+}\right)+a_{+} a_{-}\left(c_{+}^{2}+c_{-}^{2}-c_{+} c_{-}-c_{-} c_{+}\right) \\
& +a_{-} a_{+}\left(c_{+}^{2}+c_{-}^{2}+c_{+} c_{-}+c_{-} c_{+}\right) .
\end{aligned}
$$

Equating (42) and (43), we have

$$
a_{-}^{2}\left(c_{+} c_{-}+c_{-} c_{+}\right)+a_{+} a_{-}\left(-c_{-}^{2}+c_{+} c_{-}\right)+a_{-} a_{+}\left(-c_{-}^{2}-c_{-} c_{+}\right)=0 .
$$

Taking the even and odd parts of this, we have

$$
\begin{gathered}
a_{+} a_{-} c_{+} c_{-}-a_{-} a_{+} c_{-} c_{+}=0, \\
a_{-}^{2}\left(c_{+} c_{-}+c_{-} c_{+}\right)-\left(a_{+} a_{-}+a_{-} a_{+}\right) c_{-}^{2}=0 .
\end{gathered}
$$

By acting the unitary transformation $\operatorname{Ad}\left(v_{A}\right)$ on both sides of (46) where $v_{A}$ in $\mathcal{A}_{A}^{e}$ gives the implementation of $\Theta$ on $\mathcal{A}_{A}$ as (6), we have

$$
a_{-}^{2}\left(c_{+} c_{-}+c_{-} c_{+}\right)+\left(a_{+} a_{-}+a_{-} a_{+}\right) c_{-}^{2}=0 .
$$

By averaging this and (46), we have

$$
a_{-}^{2}\left(c_{+} c_{-}+c_{-} c_{+}\right)=0
$$

Similarly, we have

$$
\left(a_{+} a_{-}+a_{-} a_{+}\right) c_{-}^{2}=0 .
$$

We will see that from (45), (47), and (48), our assertion, i.e., the evenness of $\rho_{\psi_{A}}$ or (and) $\rho_{\psi_{C}}$ follows. For (47) to be satisfied,

$$
a_{-}^{2}=0 \quad \text { or/and }\left(c_{+} c_{-}+c_{-} c_{+}\right)=0 \text {, }
$$

as $a_{-}^{2} \in \mathcal{A}_{A}^{e}$ and hence $a_{-}^{2}\left(c_{+} c_{-}+c_{-} c_{+}\right)=a_{-}^{2} \otimes\left(c_{+} c_{-}+c_{-} c_{+}\right)=0$. In the same way,

$$
c_{-}^{2}=0 \quad \text { or/and }\left(a_{+} a_{-}+a_{-} a_{+}\right)=0 .
$$

If $a_{-}^{2}=0$, then $a_{-}=0$ since $a_{-}$is self-adjoint. Therefore, $\rho_{\psi_{A}}^{1 / 2}$ is even and so $\rho_{\psi_{A}}$ is. If $c_{-}^{2}=0$, then $\rho_{\psi_{C}}$ is even. We now consider the remaining possibility, i.e., the case where $a_{+} a_{-}+a_{-} a_{+}=c_{+} c_{-}+c_{-} c_{+}$ $=0$. This implies that $\rho_{\psi_{A-}}=\rho_{\psi_{C-}}=0$, namely both of $\rho_{\psi_{A}}$ and $\rho_{\psi_{C}}$ are even. In conclusion, at least one of the marginal states $\rho_{\psi_{A}}$ and $\rho_{\psi_{C}}$ should be even.

Now we know that the product state $\psi_{A}{ }^{\circ} \psi_{C}$ exists and can use the argument in (32) that leads to our desired assertion.

We shall go back to three-composed systems and comment on the condition of the strong additivity of entropy. For now, we are only able to produce the desiarble form of Markov property for even states. We guess that the assumed strong additivity of entropy may control in a certain sense nonevenness of the states satisfying this as for the case of two-composed systems above. Without the evenness assumption, we need more involved analysis to understand the structure of those states satisfying the strong additivity of entropy. 


\section{ACKNOWLEDGMENTS}

The author thanks paticipants of the von Neumann Conference, Budapest, October 2003 and of 26th Conference of QP and IDA in Levico (Trento), February, 2005 for useful conversation.

${ }^{1}$ Accardi, L., "The noncommutative markovian property (Engl.)," Funct. Anal. Appl. 9, 1-7 (1975).

${ }^{2}$ Accardi, L., “Topics in quantum probability,” Phys. Rep. 77, 169-192 (1981).

${ }^{3}$ Accardi, L. and Cecchini, C., "Conditional expectations in von Neumann algebras and a theorem of Takesaki," Funct. Anal. Appl. 45, 245-273 (1982).

${ }^{4}$ Accardi, L., Fidaleo, F., and Mukhamedov, F., "Markov states on the CAR algebra," math-ph/0411035.

${ }^{5}$ Araki, H. and Moriya, H., "Equilibrium statistical mechanics of fermion lattice systems," Rev. Math. Phys. 15, 93-198 (2003).

${ }^{6}$ Hayden, P., Jozsa, R., Petz, D., and Winter, A., "Structure of states which satisfy strong subadditivity of quantum entropy with equality," Commun. Math. Phys. 246, 359-374 (2004).

${ }^{7}$ Jenčová, A. and Petz, D., "Sufficiency in quantum statistical inference," Commun. Math. Phys. (to be published).

${ }^{8}$ Lieb, E. H. and Ruskai, M. B., "A fundamental property of quantum-mechanical entropy," Phys. Rev. Lett. 30, 434-436 (1973).

${ }^{9}$ Moriya, H., "Some aspects of quantum entanglement for CAR systems," Lett. Math. Phys. 60, 109-121 (2002).

${ }^{10}$ Moriya, H., "Validity and failure of some entropy inequalities for CAR systems," J. Math. Phys. 46, 033508 (2005).

${ }^{11}$ Moriya, H., "On separable states for composite systems of distinguishable fermions," J. Phys. A: Mathematical and General (to be published).

${ }^{12}$ Mosonyi, M. and Petz, D., "Structure of sufficient quantum coarse-grainings," Lett. Math. Phys. 68, 19-30 (2004).

${ }^{13}$ Ohno, H., "Extendability of generalized quantum Markov states," in Quantum Probability and Infinite Dimensional Analysis from Foundations to Applications, edited by M. Schürmann and U. Franz (Krupp-Kolleg Greifswald, Germany, 2003), pp. 415-427.

${ }^{14}$ Ohya, M. and Petz, D., Quantum Entropy and Its Use (Springer-Verlag, New York, 1993).

${ }^{15}$ Petz, D., "Sufficiency of channels over von Neumann algebras," Q. J. Math. 39, 97-108 (1988).

${ }^{16}$ Petz, D., "Monotonicity of quantum relative entropy revised," Rev. Math. Phys. 15, 79-91 (2003). 